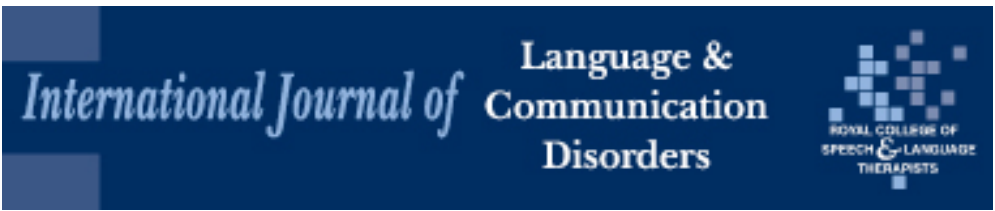

\title{
Speech and Language Therapy for Primary Progressive Aphasia across the UK: A survey of current practice.
}

\begin{tabular}{|r|l|}
\hline Journal: & International Journal of Language \& Communication Disorders \\
\hline Manuscript ID & TLCD-2019-0061.R1 \\
\hline Wiley - Manuscript type: & Research Report \\
\hline Keywords: & $\begin{array}{l}\text { Primary Progressive Aphasia, speech and language therapy, assessment, } \\
\text { treatment, survey }\end{array}$ \\
\hline
\end{tabular}

\section{SCHOLARONE \\ Manuscripts}




\title{
Full Title:
}

Speech and Language Therapy for Primary Progressive Aphasia across the UK: A survey of current practice.

\footnotetext{
Keywords: Primary Progressive Aphasia; Speech and Language Therapy; Assessment; Treatment; Survey.
}

\begin{abstract}
:
Background

Primary progressive aphasia (PPA) describes a heterogeneous group of language led dementias. People with this type of dementia are increasingly being referred to speech and language therapy services. Yet there is a paucity of research evidence focusing on PPA interventions and little is known about speech and language therapy practice in terms of assessment and provision of intervention.
\end{abstract}

Aim 
To survey the practices of speech and language therapists in the areas of assessment and intervention for people with PPA.

Methods \& Procedures

A 37-item, pilot-tested survey was distributed electronically through the Royal College of Speech and Language Therapists (RCSLT), Clinical Excellence Networks (CENs), and social media networks. Survey items included questions on care pathways, assessment and intervention approaches, and future planning. Analysis was conducted using descriptive statistics and thematic analysis.

Outcomes \& Results

One hundred and five SLTs completed the survey. Respondents reported more frequently using formal assessment tools designed for stroke related aphasia than for dementia. Informal interviews were reportedly always used during assessment by almost $80 \%$ of respondents.

Respondents were significantly more likely to use communication partner training than impairment focused interventions. Goal attainment was the most commonly used outcome measure. Respondents provided 88 goal examples which fell into six themes: 1 .

Communication Aid; 2. Conversation; 3. Functional Communication; 4. Impairment focused; 5. Specific strategy; 6. Communication Partner. Additionally, respondents reported addressing areas such as future deterioration in communication and cognition, decision making and mental capacity, and driving. Ten (9.4\%) respondents reported the existence of a care pathway for people with PPA within their service. 
Conclusions \& Implications

This survey highlights the range of current PPA assessment and intervention practices in use by the respondents. Communication partner training is commonly used by the surveyed

$\underline{\text { SLTs, }}$, despite the lack of research evidence examining its effectiveness for PPA. There is a need to develop evidence based, care pathways for people with PPA in order to advocate for further commissioning of clinical services.

\section{Background:}

Dementia has been identified as the leading cause of death in England and Wales (Office of National Statistics 2015) and it is estimated that a third of people born in the United Kingdom (UK) in 2015 will go on to develop dementia (Lewis 2015). At present frontotemporal dementia (FTD) is the most common cause of younger onset dementia with UK population prevalence estimates of approximately 11/100,000 (Coyle-Gilchrist et al. 2016). Primary Progressive Aphasia (PPA) syndromes collectively account for around a third of these cases (CoyleGilchrist et al., 2016). This conservative estimate means there are at least 2300 people currently living with PPA in the UK. 
PPA describes a heterogeneous group of language led dementias, often associated with FTD and Alzheimer's disease, which, in the initial stages, presents as an insidious deterioration in language skills, on a background of intact cognitive functions (Mesulam 1982). Currently there are three internationally recognised PPA syndromes, each presenting a distinct profile of language difficulties (Gorno-Tempini et al. 2011; Marshall et al. 2018). Semantic variant PPA (svPPA, most often associated with an underlying FTD pathology, sometimes called semantic dementia) results in difficulties in understanding word meanings. People with svPPA present with fluent, often empty speech and will have difficulty naming objects yet are able to use and understand grammar appropriately. Logopenic variant PPA (lvPPA, most often associated with an underlying Alzheimer's pathology) is identified by an individual's difficulties in expression. Although fluent, people with lvPPA present with difficulties accessing word forms and with phonological assembly. Non-fluent agrammatic variant PPA (navPPA, most often associated with an underlying FTD pathology) presents as a dysfluent apraxic disorder and/or an agrammatism. People with navPPA demonstrate groping, effortful speech, and may use increasingly less complex grammatical structures over time.

Given the centrality of communication difficulties experienced by people with PPA and their families, a referral to speech and language therapy seems warranted. Yet people with PPA report being more isolated from speech and language therapy services than from any other 
allied health discipline (Riedl et al. 2014). The authors of this German study suggest that this may be due to the rarity of the condition, resulting in relatively few people with PPA living within a speech and language therapy service's catchment area. This in turn may mean the SLTs themselves have little experience in working with people with the condition. However, in a companion paper to this one reporting different aspects of the same survey $\overline{,}_{2}$ Volkmer et al (2018) highlight a third of 105 UK SLTs working with people with PPA report an increase in referral numbers over recent years, mostly from neurologists. A total of 353 people with PPA were reported as seen over a 24-month period, averaging 3.27 per SLT respondent. In addition, more than three quarters of Volkmer et al's (2018)the respondents reported that there were people with PPA who were not able to access their services, most often due to a lack of awareness amongst referrers of the SLT's role, difficulties in diagnosis, and restrictive service criteria. Researchers in the United States also report an increase in referrals for both assessment and differential diagnosis of PPA variant, as well as for treatment (Henry \& Grasso, 2018). Taylor et al (2009) hypothesize that, in Australia, neurologists are most likely to refer to speech and language therapy as a result of knowledge of the treatment SLTs provide to people with non-progressive stroke related aphasia.

Given its degenerative nature, care pathways for PPA need to include different components than those for people with acute onset non-progressive aphasia. The European Pathway 
Association (2007) defines a care pathway as "an explicit statement of the goals and key elements of care based on evidence, best practice and patient expectation”. Volkmer et al, (2018) highlight that mMore than $90 \%$ of SLT respondents to aacross the UK who were wide survey of SLTs-working with people with PPA reported there was no care pathway for PPA within their services-(Volkmer et al, 2018). Taylor et al. (2009, p.12) reported "no clear single management pathway" for speech and language therapy practice for PPA in New South Wales, Australia.

Previous attempts to outline key elements of care for PPA have come from studies carried out in the US. For example, Rogers \& Alarcon (1998) describe three key principles for management of people with PPA including planning interventions in anticipation of continued decline, working with communication partners upon whom individuals will inevitably become dependent, and directing therapy at the level of disability since restitution is not ultimately possibly given the deteriorating nature of the disease process. In contrast, a phased approach to treatment has been proposed by Hinshelwood \& Henry (2016), whereby in the mild stage of the disease restorative therapies are employed, in the moderate stages $\mathrm{AAC}$ and multimodal communication are prioritised, and in later stages there is a focus on environmental supports and communication partner training (CPT). More recently, Rogalski and colleagues, have advocated an overarching person-centered approach that "proactively informs the care plan through dynamic interactions with the clinician" (Rogalski et al. 2016, 
p.286) in order to maximize participation in daily life activities and maintain independence (see also Rogalski \& Khayum, 2018).

\begin{abstract}
While such elements inform a PPA care pathway for speech and language therapy services, there remains a paucity of research evidence to underpin specific intervention approaches, and a lack of knowledge of best practice and client expectation. A 2013 systematic review of nonpharmacological interventions for PPA identified 39 studies investigating interventions for a total of 67 people (Carthery-Goulart et al. 2013). Twenty-one of these described interventions targeting solely svPPA and 31 of the 39 studies were impairment based interventions (word relearning). The effectiveness of restorative word retrieval therapies for PPA is the focus of
\end{abstract} tTwo more recent systematic reviews exist of the effectiveness of restorative word retrieval therapies for PPA-(Jokel et al. 2015; Cadório et al. 2017) and a research update (Croot, 2018). A systematic review of the effects of functional communication focused interventions for people with PPA and their caregivers is currently in preparation by the authors.

Despite the focus on impairment based interventions in the research evidence, limited literature on best practice indicates that functional communication focused interventions are considered a higher clinical priority (Volkmer 2013). Kindell et al (2015) conducted a study with six SLTs in England to ascertain consensus on the focus of speech and language therapy for svPPA 
(referred to as semantic dementia). The top three therapeutic priorities were enabling better communication with family carers, supporting and educating the multidisciplinary team (MDT), and focusing on abilities and strengths (Kindell et al. 2015).

This study aims to reveal the practices of UK SLTs in the areas of assessment and management of PPA, including outcome measurement. The literature suggests that SLTs have a wide ranging role in supporting people with PPA (Marshall et al. 2018; Volkmer 2013) but no-one yet has a UK-wide perspective on this.

\section{Method:}

This survey conforms to the CHERRIES survey reporting checklist (Eysenbach, 2004) and Turk et al's (2018) extension to this. The Departmental Ethics Chair in Language and Cognition at University College London waived approval as the study met the criteria for service evaluation.

Survey development

Previous speech and language therapy surveys of clinical practice provided direction on development of survey sections and questions, specifically PPA in an Australian context (Taylor et al. 2009), non-progressive aphasia (Beckley et al. 2017; Sirman et al. 2017) and 
progressive dysarthria (Collis \& Bloch 2012). Literature on speech and language interventions for PPA (Carthery-Goulart et al. 2011; Volkmer 2013; Kindell et al. 2015) was consulted to guide category fields for questions related to assessment and intervention. Occupational and geographical domains were based on recognised socio-economic classifications (Office for National Statistics 2010) and statistical regions (Office for National Statistics 2009) within the UK. Questions were refined by the first author (an SLT) with expert advice from co-authors to ensure relevance, until a 37-item survey was agreed for initial piloting.

\section{Pilot phase}

A convenience sample of six practising SLTs piloted the survey to assess usability. To maintain the largest possible sample for the main study, we invited SLTs based in the UK who do not work with people with PPA, and SLTs who see people with PPA but work in Australia (this was a convenience sample of SLTs known to the first author). Pilot respondents recorded the time taken to complete the survey and provided written feedback on the format, category fields and wording of questions. Modifications in the light of this feedback included introduction of a number of 'other' or 'none' categories and provision of category examples in one question. Additionally, the survey format was modified to ensure respondents had to provide an answer before moving on, and a 'back' button was inserted so respondents could review their answers. 
After piloting, the number of questions in the final survey was unchanged. Questions were not randomised, and page numbers not displayed, instead a progress bar was provided.

The final survey comprised five sections: 1) Clinical background; 2) Number, type and source of patients; 3) Time spent on management of people with PPA; 4) Specific assessment and intervention approaches; 5) Outcome measures and planning for the future. There were closed questions (for speed of completion) and open questions to elicit additional information (Schaeffer \& Dykema 2011). The complete survey is presented in Appendix 1.

Main survey

This survey was delivered online to facilitate dissemination across the UK. It was designed and made epenly available to respondents, for 8 weeks from the $4^{\text {th }}$ February to the $7^{\text {th }}$ April 2016, using Opinio 7.3 software. Once closed, no further responses were recorded. The initial page of the online survey provided a statement making clear that by continuing, respondents were giving consent to participate in the survey and that their responses would be stored anonymously. No personal data was collected.

Sample selection 
In order to achieve a representative cohort of UK SLTs, the Royal College of Speech and Language Therapists (RCSLT) was contacted to facilitate survey dissemination. A letter to the editor containing the survey link was published in the RCSLT practice magazine, 'The Bulletin', which is distributed to all 13,809 practising members. The survey link was also disseminated through an RCSLT electronic research newsletter and was emailed to 14 relevant Clinical Excellence Networks associated with the RCSLT for onward dissemination to members. Email and social media were also used to disseminate the link through the first and fourth authors' networks, including via Twitter and the first author's blog site. A reminder was sent via all these routes 2 weeks prior to closing the survey (see supplementary information for dissemination details). The email inviting SLTs to take part mentioned the development of an intervention but did not refer to CPT.

Participant inclusion criteria comprised SLTs a) practising in the UK (practising SLTs are employed in the UK National Health System from a band 5; entry level, to band 8; highly specialist, manager or consultant) and b) who had ever worked with a person with diagnosed or possible PPA. Respondents completed questions in section 1 of the survey on qualification, years of employment, and experience of working with people with PPA. If they did not meet the inclusion criteria at this point they exited the survey. Respondents who met the study criteria continued to section 2 of the survey. 


\section{Data analysis}

Following the elimination of duplicates (the last entry was used), completed surveys were extracted and analysed. Data were downloaded to IBM's Statistical Package for Social Science 23 software. Response rates were calculated in accordance with the CHERRIES definition (Eysenbach, 2004). Data on referral rates and patterns, and barriers to accessing speech and language therapy are reported in Volkmer et al (2018). This article reports on care pathways, assessment and intervention practices, outcome measurement, and future planning. A chi square test of independence was performed to examine the relation between intervention type and frequency of use. Remaining data were examined using frequency counts and contingency tables (Sauerbrei \& Blettner 2009). For open ended questions or 'other' response categories, both descriptive statistics, such as coding and counting responses, and qualitative analysis was used. The latter included thematic analysis (Braun \& Clarke 2006) of intervention goals supplied by respondents.

\section{Results}

\section{Demographics of SLT respondents}

There were 179 unique survey visitors of which 106 agreed to participate by starting to complete the survey, resulting in a participation rate of 179:106. One participant exited the 
survey because they had insufficient experience with PPA. The remaining $O$ f these, 105 hadd

experience of working with people with PPA and submitted complete surveys, resulting in a completion rate of 106:105. There is no available data on numbers of UK SLTs providing services for PPA, therefore it is not possible to calculate the percentage response rate.

Respondents were from a range of grade bands between 5 and 8, and worked across several professional locations, i.e. primary care, acute health and mental health. Table 1 summarises respondent characteristics. Just over a third were employed at band $7(37.1 \%, n=39)$ and just under a third at band 6 level $(31.4 \%, n=33)$. More than half had over 10 years' experience $(54.3 \%, \mathrm{n}=57)$. Just under half were based in primary care $(45.7 \%, \mathrm{n}=48)$, with 28 in acute health $(26.7 \%)$ and 15 in mental health care $(14.3 \%)$. There is no national data on proportions of SLTs employed in primary care, acute health and mental health, yet this sample appears representative as services to mental health are known, within the profession, to be less.fewer. On comparing respondent rates across UK regions (Office for National Statistics 2009) the highest number of respondents were from Scotland $(17.1 \%, \mathrm{n}=18)$ and fewest respondents came from the North West of England $(1.9 \%, n=2)$.

INSERT TABLE 1: Characteristics of SLT survey respondents

Care Pathways 
All respondents who reported having a care pathway for people with PPA $(9.4 \%, n=10)$ were asked to describe this pathway (see Figure 1 for key components). One respondent listed an onward referral process to another SLT service. Two respondents described their service's care pathway as comprising only assessment and advice. One respondent provided a detailed seven stage care pathway delivered over six sessions, where following assessment at stage 1 , the remaining stages focused on a range of interventions including "Rehearsal and integration of appropriate strategies into communication, through modelling, rehearsing and home tasks which incorporate significant communication partners. Provision of information regarding appropriate high-tech communication aids". This respondent was the only one who referenced the intervention research literature. The other six respondents provided an overview of their service's care pathways by giving some examples of intervention mode (individual, dyadic \& group) or activities that may be offered. Only one of the 10 respondents reporting a local PPA care pathway described dysphagia assessment and management.

Of the 10 , only one respondent described being involved in the diagnosis of PPA, although seven respondents stated that assessment was part of the care pathway. When describing the assessment stage of the care pathway, one respondent provided detailed information including tools used:

\footnotetext{
"Assessment of impairment and activity/participation. This will include an aphasia and communication strengths and weaknesses profile, with specific consideration of
} 
spontaneous strategy use by the client. It will also include identification of the client's significant communication partners and their roles. (Assessments: dynamic testing with Boston Naming Test (BNT); PALPA subtests; CAT subtests).”

Seven of the 10 respondents with a care pathway stated that intervention was a part of it, with two explaining that this was based on the "individual's profile". Three respondents mentioned impairment focused interventions, with one of the three respondents describing the provision of:

\author{
"Impairment based neuro-protective therapy drilling of functional words (written \\ and/or spoken as appropriate) through modelling in session and in home- tasks. Control \\ items to be used to aid evaluation of this input."
}

Another respondent added the caveat that:

"A package of impairment-based therapy may be offered and provided if the client is keen, has support and responds well”.

Education and "explanation of the condition" was mentioned by three respondents as part of the intervention stage of their service's care pathway. Three respondents described provision of group therapy (one of whom specified a couples group), and six respondents described involvement of a carer. 
Functional communication focused intervention options were described by four respondents, with one commenting:

“Training in supportive conversational partner strategies offered (not formal training package, but tailored to individual). Low-tech AAC \& communication passports/life story work".

Finally, six of the 10 respondents with a care pathway described ongoing or future input such as onward referral to other services, signposting to support groups, and review or maintenance sessions within the speech and language therapy service.

INSERT FIGURE 1. Components of care pathways described by SLT respondents

All respondents were asked how many sessions they devoted to assessment and management activities. Respondents were given the following definitions: Assessment includes assessment of language and communication, contributing to diagnosis of PPA, assessment of dysphagia and mental capacity assessment; Management includes case/care review meetings, joint sessions with other disciplines, education for person with PPA, family education, staff education, impairment-focused language intervention, functional communication focused interventions, combined language/communication focused intervention, group therapy with people with PPA, management of dysphagia. Figure 2 summarises the average number of SLT sessions respondents reportedly spent on assessment and management activities. On average, 
respondents spent 2.2 sessions on communication assessment, but only 1.2 sessions on diagnosis, 0.8 sessions on dysphagia assessment and 0.5 sessions on mental capacity assessment. Results show that respondents spent more time on functional communication focused interventions than on any other activity, with an average of 4.9 sessions. This contrasts with 2.8 sessions for impairment focused intervention, 2.8 sessions for combined language \& communication intervention, 2 sessions on family education, 2 sessions on education for the person with PPA, and 1.5 sessions on group therapy. The smallest amount of time was spent on joint sessions with other disciplines, on average 0.5 sessions.

INSERT FIGURE 2. Average number of sessions spent on assessment and management activities by SLT respondents working with people with PPA across the UK

\section{Assessment Tools}

Respondents were asked to rate on a 5-point scale (never, occasionally, sometimes, often, always) the frequency with which they used a selection of 10 listed assessment tools over the last 24 months. Figure 3 presents the reported frequency of use of assessment tools. The least used tool, Mini Mental State Exam (MMSE; Creavin et al. 2016), was rated as never used by $83 \%(n=87)$ of respondents and always used by $1.9 \%(n=2)$ of respondents. Comparatively the most used tool, informal interview, was rated as never used by $4.7 \%(n=5)$ respondents and 
always used by $78.1 \%(\mathrm{n}=82)$ of respondents. The second most commonly used tool, informal functional assessment was rated as never used by $6.67 \%(n=7)$ of respondents and always used by $65.7 \%(n=69)$ of respondents. The third most commonly used tool, informal impairment based communication screen, was rated as never used by $15.2 \%(n=16)$ and always used by $38.1 \%(n=40)$. The most commonly used formal published assessment was identified as the Comprehensive Aphasia Test (CAT; (Swinburn, Porter and Howard, 2004), always or often used by $44 \%$ of $(n=46)$ respondents and never used by $30.5 \%(n=32)$ of respondents. When asked to state other assessments they used, respondents identified 28 further formal tools. The most commonly named were the Mount Wilga High Level Language Test (Christie, Clark, \& Mortensen 1986) (mentioned by 6 respondents), the Montreal Cognitive Assessment (MOCA; Freitas et al. 2012) (5 respondents), the Butt Non Verbal Reasoning Test (Butt \& Bucks, 2017) (3 respondents) and the Controlled Oral Word Association Test (COWAT, Patterson 2011) (3 respondents). Of the remaining 24 stated tools, four were suggested by two respondents each, and the remainder by one respondent each.

INSERT FIGURE 3: Frequency of reported use of assessment tools with people with PPA over the last 24 months.

INSERT FIGURE 4: Frequency of reported use of intervention approaches with people with PPA over the last 24 months 


\section{Intervention approaches}

Respondents were asked to rate on a 5-point scale the frequency (never, occasionally, sometimes, often, always) with which they had used seven listed intervention approaches over the last 24 months. Figure 4 presents the reported frequency of use of intervention approaches. The least used intervention approach, impairment directed interventions for speech production, was rated as never used by $69.5 \%(n=73)$ of respondents. No respondents indicated they always used this approach but it was often used by $8.6 \%(n=9)$ of respondents. Comparatively the most used intervention, communication training for families and carers, was rated as never used by $1.9 \%(n=2)$ and always or often used by $84.8 \%(n=81)$ of respondents. The second most commonly used intervention, functional communication focused interventions, was rated as never used by $17.1 \%(n=18)$ and always or often used by $42.9 \%(n=45)$ of respondents and sometimes used by $28.6 \%(n=30)$ of respondents. Communication training for staff was rated as never used by $37.1 \%(n=39)$ respondents, always used by $20 \%(n=21)$, and sometimes used by $29.5 \%$ (31). Impairment directed interventions were rated as never used by $30.5 \%(n=32)$, always or often used by $20 \%(n=21)$, and sometimes used by $31.4 \%(n=33)$. A chi square test of independence was performed to examine the relation between intervention type (communication training/impairment focused) and frequency of use (always/never). The relation between these variables was significant, $\mathrm{X}^{2}(2, \mathrm{~N}=215)=71.6442, p=0.00$, demonstrating that communication training is significantly more likely to be rated as always used than impairment focused intervention. When asked to state other intervention approaches 
they used, respondents reported 12 options. The most commonly identified were communication books and wallet cards, mentioned by 14 respondents, whilst life story work and total communication strategies were each suggested by three respondents.

\section{Measuring outcomes}

- When asked which outcome measures they used to document progress in therapy respondents were able to choose multiple options from a list of six options. Goal setting was reported as the most commonly used measure $(53.8 \%, n=57)$, followed by selfrating scales completed by the person with PPA or their communication partner $(50 \%$, $\mathrm{n}=53)$, language assessment $(48.1 \%, \mathrm{n}=51)$, Therapy Outcome Measures (TOMS; Enderby et al. 2013$)(34.9 \%, \mathrm{n}=37)$, rating scales completed by the SLT $(19.8 \%, \mathrm{n}=21)$ and video-recording of conversation $(7.6 \%, n=8)$ (the exact aspect of the recording that provided an outcome measure is not known, since we did not ask respondents to provide further information). Respondents were asked for examples of other outcome measures they used and provided nine further tools. Audio recording, and the East Kent Outcome Measure (EKOS; Murphy \& Logan 2009) were each identified by two respondents, whilst the remaining seveneven measures: Aphasia Impact Questionnaire-21 (Swinburn 2013), client_feedback, or carer feedback, informal naming assessment, Communication Outcomes after Stroke (Long et al. 2008), Cognitive Linguistic Quick Test (Helm-Estabrooks 2001) and Visual Analogue Self-Esteem Scale (Brumfitt \& 
Sheeran 1999), were identified by only one respondent each. Respondents were asked to provide examples of the types of goals set and provided 88 in total. Six main-separate main themes arose from analysis of these goals: 1. Communication Aid; 2. Conversation; 3. Functional communication; 4. Impairment focused; 5. Specific Practising a strategy; 6. Communication Partner. Figure 5 provides examples of the goals listed by respondents. Each goal was assigned to one theme. Of the 88 goals, $50 \%$ $(n=44)$ fell into themes 2. Conversation or 3. Functional communication. Conversation goals centred around using specific strategies, such as gesture or drawing, in conversations. Fourteen of the functional communication goals related to completing specific functional communication activities with family and friends, such as ordering a meal or coffee. Thirty percent $(n=29)$ of goals focused on using a communication aid (theme 1), such as tablet devices and wallet cards or communication books in a specific situation. Only $10 \%(n=9)$ of goals fell into theme 4. Impairment focused, which comprised those associated with word relearning and naming, such as learning a certain number of words in a certain time frame. Sixteen percent $(n=14)$ of goals fell into theme 5. Practising a strategy, such as learning to use circumlocution effectively when experiencing a word finding difficulty. Finally, 9\% $(\mathrm{n}=8)$ of goals targeted a communication partner and focused on using a specific strategy to support or enable their partner with PPA in conversations. 
INSERT Figure 5. Example goals given by respondents organised by theme

\section{Future planning and onward referral}

Respondents were asked whether they addressed cognitive changes and deterioration in communication, legal issues including assessment of capacity, driving, family care supports, or family finance supports in planning for the future with people with PPA (see Table 24). Almost all respondents $(85.9 \%, n=91)$ reported that they explicitly addressed both cognitive changes and deterioration in communication. When asked to give other examples of what they addressed when planning for the future, respondents identified the following additional issues: educating children; supporting other staff; housing; signposting carers to organisations for social and emotional support; advance care planning. Respondents reported the most commonly used third sector organisations for onward referral to be the Alzheimer's Society $(51.9 \%, n=55)$ and the Rare Dementia PPA Support Group $(28.35 \%, n=30)$. Local aphasia and memory groups, dementia cafes and other dementia groups were listed, as well as a variety of organisations such as Befriending, Age UK, Age Northern Ireland, Alzheimer's Scotland, Motor Neurone Disease Scotland, Young People with Dementia Groups, and Admiral Nursing.

INSERT: Table 2: Future planning and onward referral

\section{Discussion}


The role of SLTs in PPA is an emerging area of practice across the UK. This survey highlights the range of current assessment and intervention practices of SLT respondents working with people with PPA. Importantly these results demonstrate that the range of interventions used by $\underline{\text { respondent SLTs extend beyond those with a developed evidence base for PPA. The surveyed }}$ SLT $\underline{s} s$ report that they prioritise communication training for families, suggesting that the needs of people living with PPA and their families are not being met by a focus on impairment alone.

Care pathways have a positive impact on the way care is organised (Seys et al. 2017). At present, there are no national care pathways for people with PPA or FTD, and only a small number of respondents reported having PPA care pathways within their services. A defining characteristic of a care pathway is identification of the required healthcare resources (The European Pathways Association, 2007). Of the pathways that were described by respondents, only seven of the 10 included both assessment and intervention, and only one respondent outlined a detailed multi-stage-care pathway linked to the current research evidence. Without this kind of care pathway in place, SLTs may find it difficult to commission and thus deliver best practice care for people with PPA.

Despite there being a number of dementia specific formal assessment tools available, the most popular assessments that respondents reported on were informal interviews, functional 
observation, and a stroke aphasia language battery (the CAT, Swinburn, Porter, Howard 2004). Henry \& Grasso (2018) observe that standard aphasia batteries developed for use with stroke related aphasia are better able to characterize the language profile of an individual with PPA, and to distinguish between PPA variants, in order to plan interventions, whilst assessments of dementia are generally designed to assess changes in cognition rather than language. Notably Henry \& Grasso (2018) point out that a number of tools to assess language in PPA and support intervention planning are only available in journal articles or by contacting university departments in the US and Australia. SLTs working in clinical settings in the UK are often unable to access peer reviewed articles, unless their employer subscribes to the relevant journal, which may account for these respondents' overwhelming preference for informal assessment tools. This may indicate that SLTs are unsure of what formal assessment are best to use with this client group. Alternatively, this may illustrate the need for more available assessment tools designed for people with PPA that support both diagnostic profiling and intervention planning.

The average number of sessions available for impairment focused interventions (2.8), reported by respondents, is significantly less than the number of sessions reported to be effective in a recent review of impairment-based interventions for PPA (Cadório et al. 2017). This review examined evidence from studies delivering interventions over a range of between 5 and 96 therapy sessions. Despite there being little indication of the 'optimal' dose, longer interventions did result in better outcomes in people with svPPA (Cadório et al. 2017). It is possible that 
these SLT respondents deprioritise impairment-based interventions knowing they are unable to deliver them at the intensity reported in the research literature. In-contrastdeed-80\% of SLT respondents identified that they would never or only occasionally use impairment focused interventions for speech production, and $48.6 \%$ would never or only occasionally use impairment focused interventions for naming. The disparity between recommended and clinically delivered dosages is also a known issue in stroke aphasia interventions (Doogan et al. 2018).

Clinical commissioning of healthcare in England is based on evidence produced in scientific research as well as local data, expertise and experiences (Swan et al. 2017). This approach to commissioning may account for some of the diversity in the amount of therapy that is reportedly available from SLT respondents to this survey; some services may be commissioned on expertise to which other commissioning groups do not have access. If occupational therapy or social care services for PPA are not commissioned, then an SLT may be the only health professional involved in a person's care. Consequently, these SLT respondents may prioritise broader issues such as future planning, thus reducing the time available for speech and language interventions. 
When selecting intervention approaches for people with PPA, $84.8 \%$ of survey respondents reported that they always or often choose communication partner training (CPT) for family and

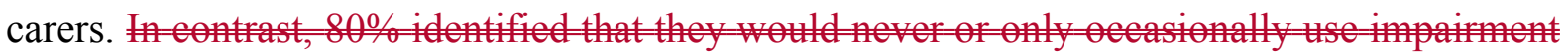
foused interventions for spech production, and $48.6 \%$ ould never only ocasionally use Similarly, 37 of 88 goals listed by survey respondents focused either on conversation or communication with family and friends, or on engaging in functional activities with family and friends. This emphasis on enabling better communication between the person with PPA and their family carer aligns with the consensus results of Kindell et al (2015), which the authors describe as "carer-focused person centred" dementia care. Rogalski and Khayum (2018) explain that the core elements of a personcentered approach to PPA include working with a client in order to facilitate their participation in everyday activities. Communicating with family and friends is a crucial everyday activity. As yet, there is no research demonstrating the effectiveness of this approach for people with PPA and their families (Taylor-Rubin et al. 2017).

\section{Implications}

This survey suggests that as a profession we need to investigate the potential to develop a care pathway for PPA in our services, then to shape this pathway to bring together scientific research, best practice and service user priorities in order to influence health commissioning. This study presents national data that SLTs can use in support of this aim. Given how many SLTs in this 
survey report using essentialcommunication training is for people with PPA and their families, demonstrating its effectiveness is also of high priority for the speech and language research community. This evidence will in turn support future endeavours to influence a national care pathway for PPA, which will require rigorous evidence from the highest levels of evidence to inform recommendations.

\section{Limitations}

It is difficult to assess how many SLTs across the UK received this survey. The RCSLT estimates that 2,000 of its registered members work with adults but there is no data on client groups, which could include, amongst others, head and neck cancer, voice, stroke, and mental health. This figure also excludes SLTs not registered with RCSLT (this is not obligatory for employment in the NHS), who may be working in private or third sector organisations. Yet, with a sample size of 105 , representing all geographical regions of the UK, the number of respondents to this survey compares favourably to other surveys of speech and language therapy practice, with respondent numbers ranging between 13 and 147 (Taylor et al. 2009; Collis \& Bloch 2012; Beckley et al. 2017; Miller \& Bloch 2017; Sirman et al. 2017). A further limitation includes access to the survey software, with some SLT respondents reporting that this was blocked by local IT services. This may have prevented some SLTs from responding. 


\section{Conclusion}

This survey has highlighted a range of assessment tools and intervention practices used by SLT respondents working with people with PPA. The-SLT respondents in this study preference for usingthe use of CPT over other interventions, despite the lack of research evidence examining its effectiveness for PPA. This approach appears to complement other aspects of a potential care pathway for PPA, such as managing cognitive and communication deterioration, and issues related to future decision making., emphasises that this should be a research priority for the profession. Importantly this study has highlighted the need for evidence based care pathways to guide SLTs working in this emerging area of practice. Care pathways can enable SLTs to advocate for commissioning of appropriately resourced services.

\section{References:}

European Pathway Association., E.P., 2007. EPA definition of Care. Available at: http://e-pa.org/care-pathways/ [Accessed August 20, 2007].

Beckley, F., Best, W. \& Beeke, S., 2017. Delivering communication strategy training for people with aphasia: what is current clinical practice? International Journal of Language \& Communication Disorders, 52(2), pp.197-213. Available at: http://doi.wiley.com/10.1111/1460-6984.12265.

Braun, V. \& Clarke, V., 2006. Using thematic analysis in psychology Using thematic analysis in psychology. Qualitative Research in Psychology, 3(2), pp.77-101. Available at: http://www.tandfonline.com/action/journalInformation?journalCode=uqrp20\%5Cnhttp:/ 
/www.tandfonline.com/loi/uqrp20\%5Cnhttp://dx.doi.org/10.1191/1478088706qp063oa.

Brumfitt, S.M. \& Sheeran, P., 1999. The development and validation of the Visual Analogue Self-Esteem Scale (VASES)1. British Journal of Clinical Psychology, 38(4), pp.387400. Available at: http://doi.crossref.org/10.1348/014466599162980.

Butt, P and Bucks, R., 2017. BNVR: The Butt Non-Verbal Reasoning Test: The Butt NonVerbal Reasoning Test., Routledge.

Cadório, I. et al., 2017. Generalization and maintenance of treatment gains in primary progressive aphasia (PPA): a systematic review. International Journal of Language and Communication Disorders, 52(5), pp.543-560.

Carthery-Goulart, M.T. et al., 2013. Nonpharmacological interventions for cognitive impairments following primary progressive aphasia A systematic review of the literature. , 7(1), pp.122-131.

Carthery-Goulart, M.T., Graham, N. \& Bak, T., 2011. Language comprehension in progressive aphasias and atypical parkinsonian syndromes. Alzheimer's and Dementia, 1), p.S752. Available at: http://ovidsp.ovid.com/ovidweb.cgi? $=\mathrm{JS} \& \mathrm{CSC}=\mathrm{Y} \& \mathrm{NEWS}=\mathrm{N} \& \mathrm{PAGE}=$ fulltext\&D $=\mathrm{e}$ med13\&AN=70502847.

Christie, J., Clark, W. \& Mortensen, L., 1986. Mount Wilga High Level Language Test, Sydney: Mt Wilga Rehabilitation Centre.

Collis, J. \& Bloch, S., 2012. Survey of UK speech and language therapists' assessment and treatment practices for people with progressive dysarthria. International Journal of Language \& Communication Disorders, 47(6), pp.725-737.

Coyle-Gilchrist, I.T.S. et al., 2016. Prevalence, characteristics, and survival of frontotemporal lobar degeneration syndromes. Neurology, 86(18), pp.1736-1743. Available at: http://www.neurology.org/lookup/doi/10.1212/WNL.0000000000002638.

Creavin, S.T. et al., 2016. Mini-Mental State Examination (MMSE) for the detection of dementia in clinically unevaluated people aged 65 and over in community and primary care populations. The Cochrane database of systematic reviews, 1, p.CD011145. Available at: http://www.ncbi.nlm.nih.gov/pubmed/26760674 [Accessed January 14, 2016].

Doogan, C. et al., 2018. Aphasia Recovery: When, How and Who to Treat? Current Neurology and Neuroscience Reports, 18(12), p.90. Available at: $\mathrm{http}: / /$ link.springer.com/10.1007/s11910-018-0891-x.

Enderby, P., John, A. \& Petheram, B., 2013. Therapy outcome measures for rehabilitation professionals: speech and language therapy, physiotherapy, occupational therapy., England: John Wiley \& Sons. 
Freitas, S. et al., 2012. Montreal Cognitive Assessment (MoCA): validation study for frontotemporal dementia. Journal of Geriatric Psychiatry \& Neurology, 25(3), pp.146154.

Gorno-Tempini, M.L. et al., 2011. Classification of primary progressive aphasia and its variants. Neurology, 76(11), pp.1006-1014.

Henry, M.L. and Grasso, S.M., 2018, July. Assessment of individuals with primary progressive aphasia. In Seminars in speech and language, 39(3), pp. 231-241.

Helm-Estabrooks, N., 2001. Cognitive linguistic quick test: CLQT., The Psychological Corporation.

Hinshelwood, H. \& Henry, M., 2016. Helping Them Hold On. ASHA Leader, 21(10), p.44. Available at:

http://leader.pubs.asha.org/article.aspx?doi=10.1044/leader.FTR1.21102016.44.

Jokel, R. et al., 2015. Word retrieval therapies in primary progressive aphasia Word retrieval therapies in primary progressive aphasia. , 7038(October).

Kindell, J., Sage, K. \& Cruice, M., 2015. Supporting communication in semantic dementia: clinical consensus from expert practitioners. Quality in Ageing and Older Adults, 16(3), pp.153-164. Available at: http://www.emeraldinsight.com/doi/full/10.1108/QAOA-082014-0016 [Accessed May 23, 2016].

Lewis, F., 2015. Estimation of future cases of dementia from those born in 2015. Alzheimers Research UK, 44(July), pp.1-12.

Long, A. et al., 2008. Development of a reliable self-report outcome measure for pragmatic trials of communication therapy following stroke: the Communication Outcome after Stroke (COAST) scale. Clinical Rehabilitation, 22(12), pp.1083-1094. Available at: http://journals.sagepub.com/doi/10.1177/0269215508090091.

Marshall, C.R. et al., 2018. Primary progressive aphasia: a clinical approach. Journal of Neurology, (0123456789). Available at: http://link.springer.com/10.1007/s00415-0188762-6.

Mesulam, M.M., 1982. [Slowly progressive aphasia without dementia]. Ann Neurol, 11, pp.592-596. Available at: http://www.ncbi.nlm.nih.gov/pubmed/2046793.

Miller, N. \& Bloch, S., 2017. A survey of speech-language therapy provision for people with post-stroke dysarthria in the UK. International Journal of Language \& Communication Disorders, 52(6), pp.800-815. Available at: http://doi.wiley.com/10.1111/14606984.12316.

Murphy, A. \& Logan, P., 2009. An analysis of the East Kent Outcome System for use in intermediate care. International Journal of Therapy and Rehabilitation, 16(9), pp.482- 
Rogalski, E. et al., 2016. Communication bridge: An internet-based person centered intervention for improving access to care and quality of life for individuals with dementia. Journal of Neurochemistry, 138, pp.422-423. Available at: http://ovidsp.ovid.com/ovidweb.cgi? $=\mathrm{JS} \& \mathrm{CSC}=\mathrm{Y} \& \mathrm{NEWS}=\mathrm{N} \& \mathrm{PAGE}=$ fulltext $\& \mathrm{D}=\mathrm{e}$ med18b\&AN=611856247.

Rogalski, E. \& Khayum, B., 2018. A Life Participation Approach to Primary Progressive Aphasia Intervention. Seminars in Speech and Language, 39(03), pp.284-296. Available at: http://www.thieme-connect.de/DOI/DOI?10.1055/s-0038-1660786.

Rogers, M.A. \& Alarcon, N.B., 1998. Dissolution of spoken language in primary progressive aphasia. Aphasiology, 12(7/8), pp.635-650.

Sauerbrei, W. \& Blettner, M., 2009. Interpreting Results in $2 \times 2$ Tables. Deutsches Aerzteblatt Online. Available at: https://www.aerzteblatt.de/10.3238/arztebl.2009.0795.

Schaeffer, N.C. \& Dykema, J., 2011. Questions for Surveys: Current Trends and Future Directions. Public Opinion Quarterly, 75(5), pp.909-961. Available at: https://academic.oup.com/poq/article-lookup/doi/10.1093/poq/nfr048.

Seys, D. et al., 2017. Better organized care via care pathways: A multicenter study. PLoS ONE, 12(7), pp.1-11. Available at: http://dx.doi.org/10.1371/journal.pone.0180398.

Sirman, N., Beeke, S. \& Cruice, M., 2017a. Professionals' perspectives on delivering conversation therapy in clinical practice. Aphasiology, 31(4), pp.465-494. Available at: https://www.tandfonline.com/doi/full/10.1080/02687038.2017.1278739. 
Sirman, N., Beeke, S. \& Cruice, M., 2017b. Professionals' perspectives on delivering conversation therapy in clinical practice. Aphasiology, 31(4), pp.465-494.

Swan, J. et al., 2017. Improving the capabilities of NHS organisations to use evidence: a qualitative study of redesign projects in Clinical Commissioning Groups. Health Services and Delivery Research, 5(18), pp.1-112. Available at: https://www.journalslibrary.nihr.ac.uk/hsdr/hsdr05180.

Swinburn, Kate Porter, Gillian Howard, D., 2004. Comprehensive Aphasia Test, Routledge, Psychology Press.

Swinburn, K., 2013. Aphasia Impact Questionnaire-21, London: Connect.

Taylor-Rubin, C. et al., 2017. Communication behaviors associated with successful conversation in semantic variant primary progressive aphasia. International Psychogeriatrics, 29(10), pp.1619-1632.

Taylor, C. et al., 2009. Speech pathology services for primary progressive aphasia: exploring an emerging area of practice. Aphasiology, 23(2), pp.161-174. Available at: $\mathrm{http} / / /$ search.ebscohost.com/login.aspx?direct=true \&AuthType $=\mathrm{ip}, \mathrm{shib} \& \mathrm{db}=\mathrm{jlh} \& \mathrm{AN}=1$ $05452226 \&$ site $=$ ehost-live $\&$ scope $=$ site.

Volkmer, A., 2013. Assessment and Therapy for Language and Cognitive Communication Difficulties in Dementia and Other Progressive Diseases, North Guilford: J\&R Press.

Volkmer, A. et al., 2018. Speech and language therapy for primary progressive aphasia: Referral patterns and barriers to service provision across the UK. Dementia, p.147130121879724. Available at: http://journals.sagepub.com/doi/10.1177/1471301218797240.

Table 1: Characteristics of SLT survey respondents

\begin{tabular}{|l|l|l|l|}
\hline \multicolumn{2}{|l|}{ Years post qualification } & Current banding \\
\hline $1-2$ & $12.4 \%(13)$ & Newly qualified & $5.7 \%(6)$ \\
$3-4$ & $6.7 \%(7)$ & Band 5 & $6.7 \%(7)$ \\
$4-10$ & $26.7 \%(28)$ & Band 6 & $31.4 \%(33)$ \\
$10+$ & $54.3 \%(57)$ & Band 7 & $37.1 \%(39)$ \\
& & Band 8 & $11.4 \%(12)$ \\
\hline
\end{tabular}




\begin{tabular}{|l|l|l|l|}
\hline \multicolumn{2}{|l|}{} & Other & $7.6 \%(8)$ \\
\hline Healthcare service & Geographical region \\
\hline Acute Health care & $26.7 \%(28)$ & East & $7.6 \%(8)$ \\
Mental Health care & $14.3 \%(15)$ & East Midlands & $4.8 \%(5)$ \\
Primary Care & $45.7 \%(48)$ & Northern Ireland & $9.5 \%(10)$ \\
Charity/Third sector & $3.8 \%(4)$ & London & $13.3 \%(14)$ \\
Independent/Private & 0 & North East & $3.8 \%(4)$ \\
Other & $9.5 \%(10)$ & North West & $1.9 \%(2)$ \\
& & Scotland & $17.1 \%(18)$ \\
& & South East & $15.2 \%(16)$ \\
& & South West & $10.5 \%(11)$ \\
& & Wales & $5.7 \%(6)$ \\
& & West Midlands & $4.8 \%(5)$ \\
& & Yorkshire and the & $5.7 \%(6)$ \\
& & Humber & \\
\hline
\end{tabular}

Table 2: Future planning and onward referral

Future planning and onward referral (\% response; multiple responses allowed)

Areas that SLTs explicitly address in future planning 
planning for legal, financial, health and social care issues

family care supports such as respite

driving

family, financial supports such as benefits

$15.1 \%(\mathrm{n}=16)$

Most commonly used third sector organisations for onward referral

Alzheimer's Disease Society

$51.9 \%(n=55)$

Rare Dementia PPA support group

Stroke Association

$15.1 \%(n=16)$

Connect

$13.2 \%(n=14)$

Dyscover

$3.8 \%(n=4)$ 


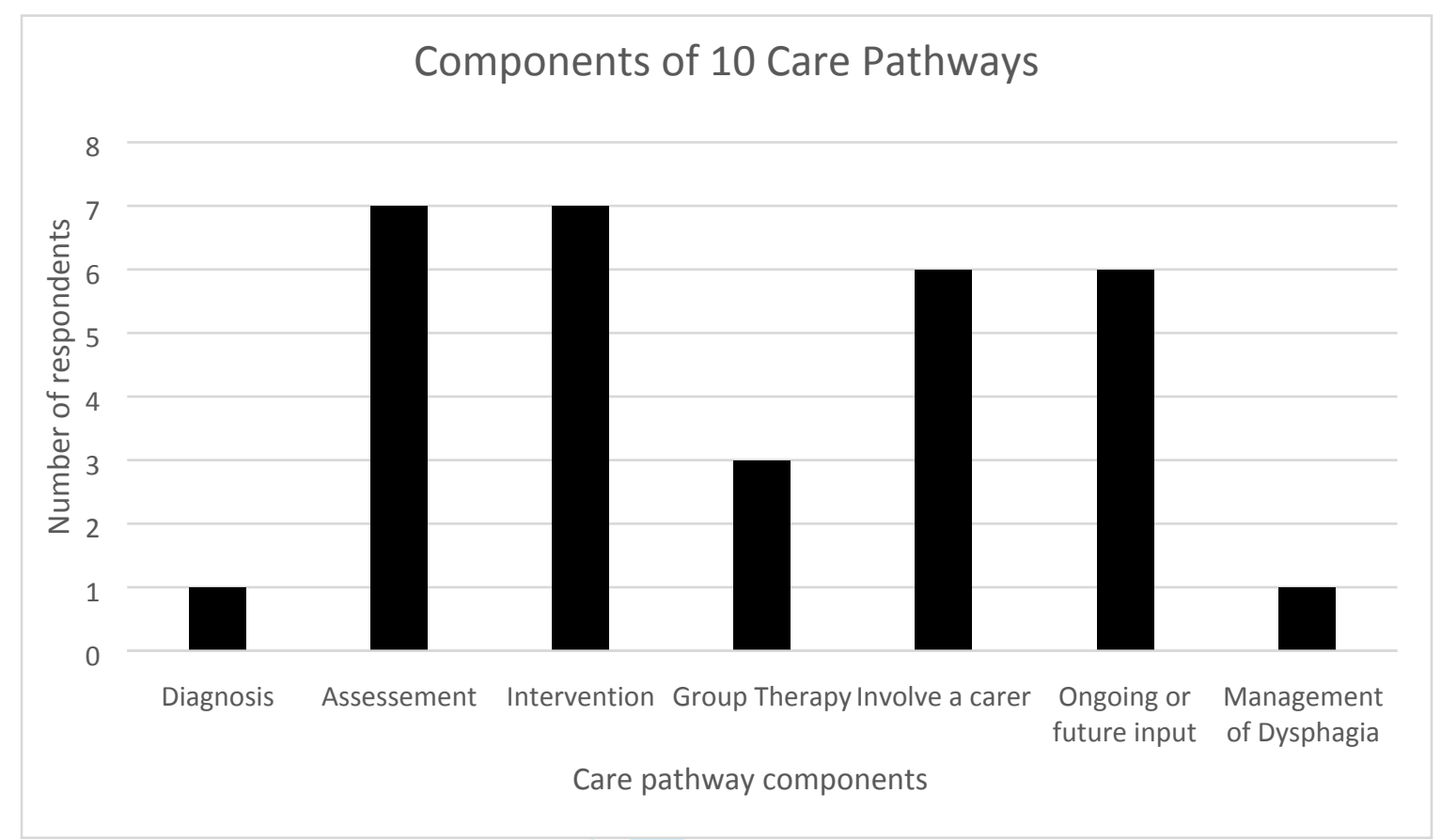

Figure 1. Components of care pathways described by SLT respondents 


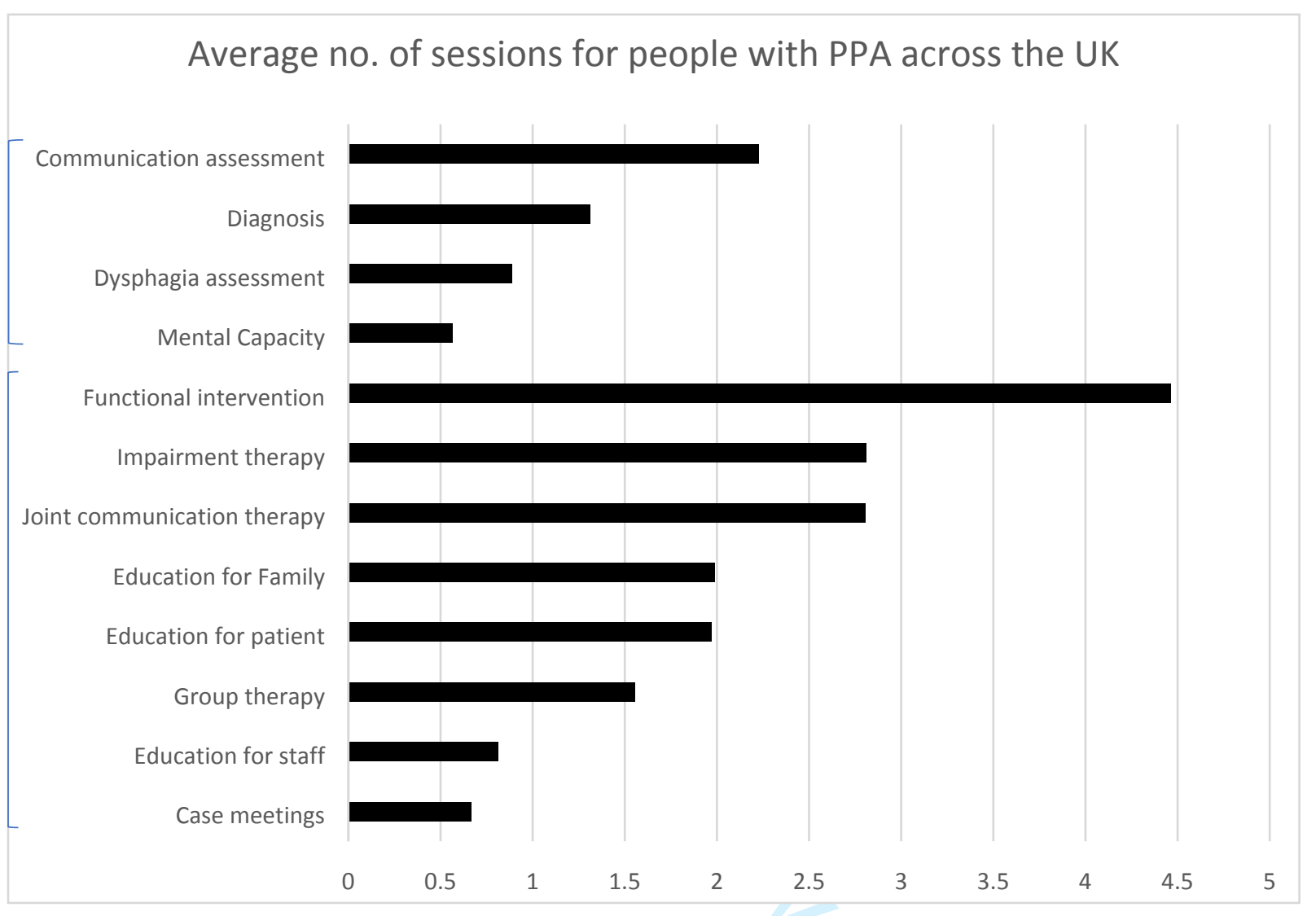

\section{Number of sessions}

Assessment activities were defined as: assessment of language and communication, contributing to diagnosis of PPA, dysphagia assessment, mental capacity assessment. Management activities were defined as: functional communication focused interventions, impairment-focused language intervention, combined language/communication focused intervention, family education, education for person with PPA, group therapy with people with PPA, staff education, case/care review meetings.

FIGURE 2. Average number of sessions spent on assessment and management activities by SLT respondents working with people with PPA across the UK 


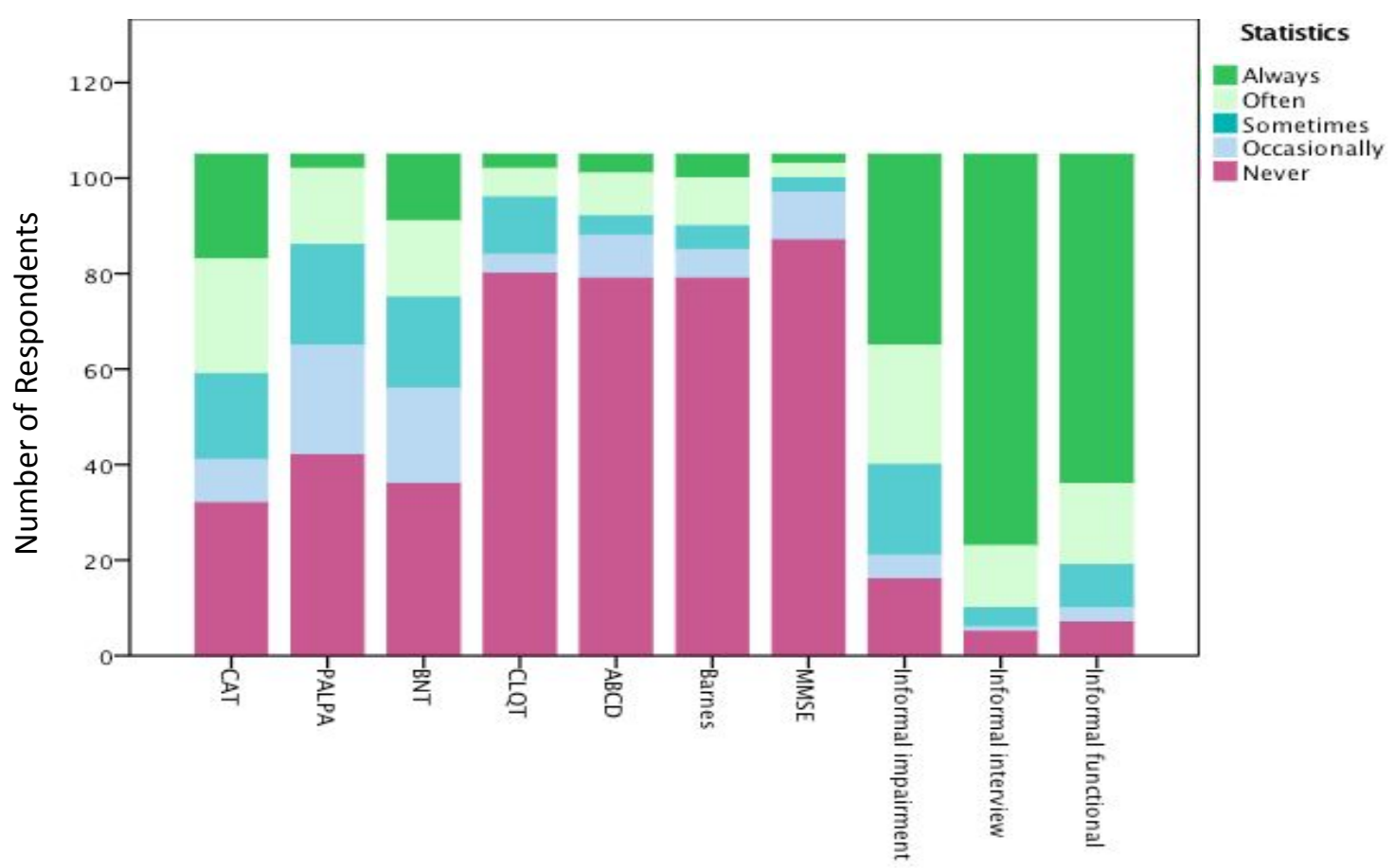

Assessment Tools

FIGURE 3: Frequency of reported use of assessment tools with people with PPA over the last 24 months. 


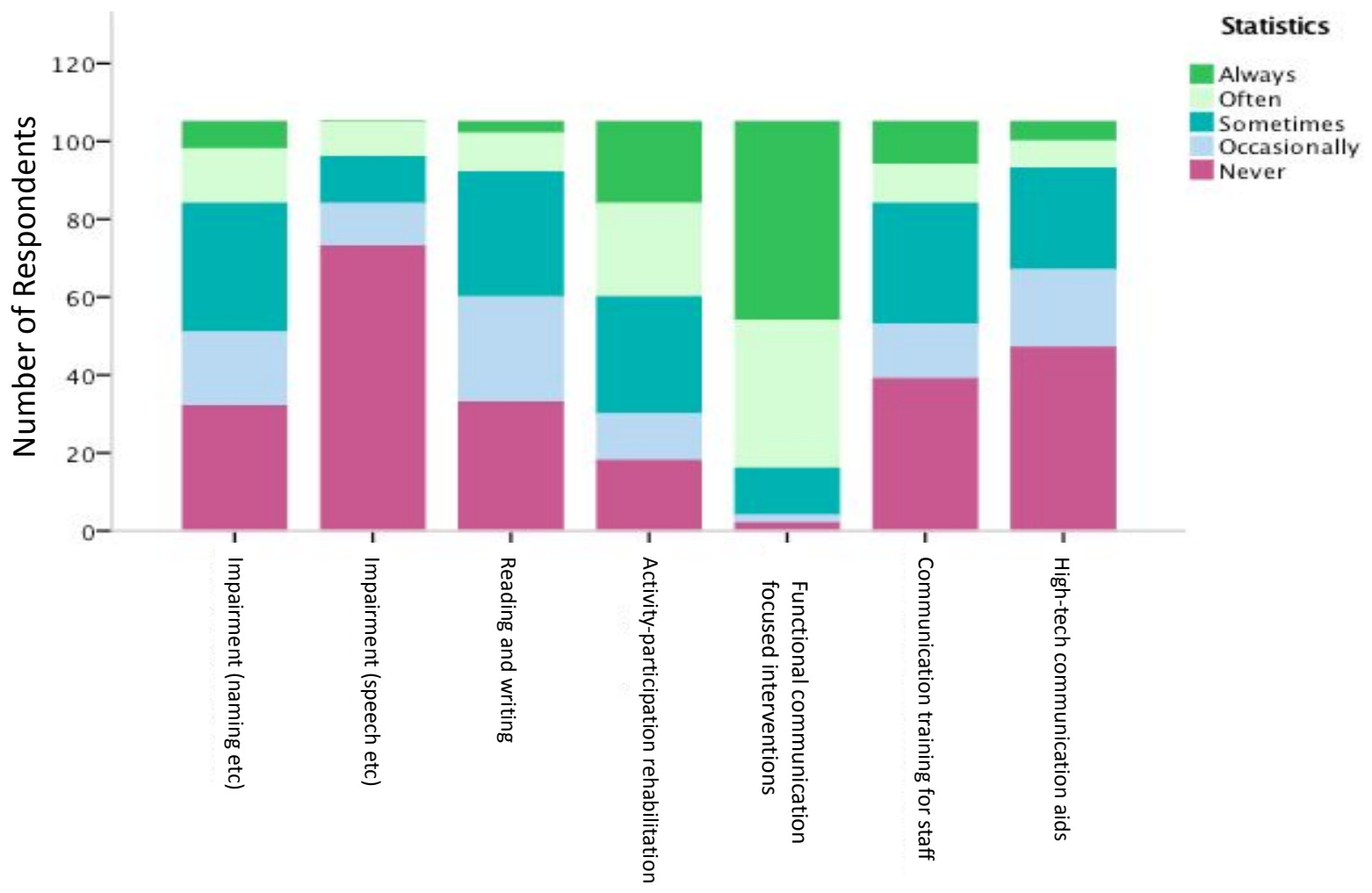

Intervention Approaches

FIGURE 4: Frequency of reported use of intervention approaches with people with PPA over the last 24 months 


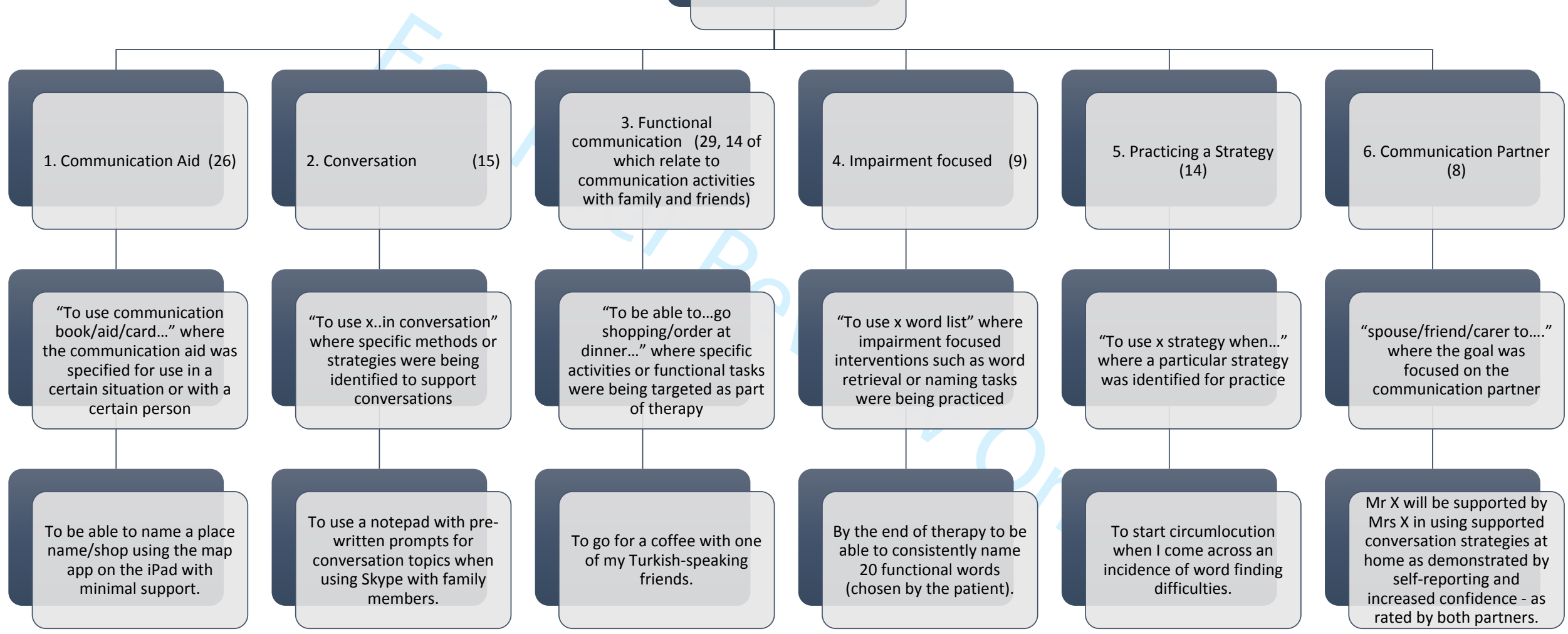

Figure 5: Example goals given by respondents organised by theme 


\section{Supplementary Material 1}

\section{Dissemination of survey via emails used the following information:}

I would be most grateful if you could disseminate the survey below to as many speech and language therapists to whom this may be relevant.

\section{Calling all Speech and Language Therapists (SLTs) who see people with Progressive Aphasia:}

PPA (a type of dementia) is a progressive language impairment. It is often diagnosed when people are in their 50s and 60s. Speech and language therapy services provided to this group are variable and anecdotally therapists report using many different approaches in managing this group.

Last summer I was awarded an NIHR Doctoral Research Fellowship to complete a PhD at UCL, supervised by Dr Suzanne Beeke and Dr Aimee Spector. My research will focus on refining and piloting an intervention for people with primary progressive aphasia (PPA).

The first stage of my research is to conduct a survey of current speech and language therapy practices across the UK. If you work with adults with neurological conditions and have seen someone with PPA in the last 24 months please complete my survey. The link is:

https://opinio.ucl.ac.uk/s?s=42060

I am currently only planning to keep the survey open for the next couple of months so please do distribute and respond if you are able.

You can follow the project on twitter @volkmer_anna or https://annavolkmersbigphdadventure.wordpress.com.

Or email me at: a.volkmer.15@ucl.ac.uk

Many thanks in advance.

Anna 


\section{APPENDIX 1: Survey Questions}

\section{Section 1: Your clinical background}

1. My job title is (open box)

2. How many years post-qualification are you? Please select one option.

Newly Qualified

$$
1-2
$$

$3-4$

4-10

$10+$

3. What is your current banding? Please select one option.

Newly Qualified Band 5

Band 5

Band 6

Band 7

Band 8

Other, please specify.

4. What healthcare service is your position funded by? Please tick one box:

Acute Health Care

Mental Health Care

Primary Care

Charity / Third sector

Independent / Private

Other, please specify

5. Which of these categories most closely represents where you work geographically?

Please tick one box:

South west

South East

London

East 
West Midlands

East Midlands

Yorkshire and The Humber

North West

North East

Ireland

Wales

Scotland

Outside the UK

6. Have you EVER seen a patient with confirmed or queried PPA?

Yes/No

[If no please finish the survey now and enter submit]

7. Which setting have you seen patients with PPA in? Please tick all those that apply.

Acute Medical

Inpatient Rehabilitation Unit

Outpatients

Community- domiciliary, ESD

Memory Clinic

Other, please specify

\section{Section 2: Number of patients, types of patients and where from?}

8. Please estimate how many patients with PPA you have had on your caseload over the last 24 months? (please indicate a specific number)

9. Please estimate the age range of the patients referred:

$<50$ years of age

50-60 years of age

60-70 years of age

$>70$ years of age

10. Please estimate what percentage came from the following backgrounds? 
English speaking background only

Non-English speaking background

$\mathrm{Bi} /$ Multi-lingual background

If you saw individuals who spoke languages other than English, please list below the languages used:

11. Of the patients referred to you with PPA what were their current or previous professions:

(Please estimate what percentage were from the following professional areas)

Higher managerial, administrative \& professional occupations

Intermediate occupations (such as supervisory, clerical \& junior managerial, administrative, professional occupations)

Routine and manual occupations (such as skilled, semi-skilled \& unskilled manual occupations)

Never worked or long-term unemployed

12. Do you feel there are people with PPA who haven't accessed the services you provide?

Yes/No

If yes what are some of the barriers (please tick all that apply):

Geographical location

Language barrier

Offered by decline

Service criteria

Other: (please explain)

13. Compared to previous years do you feel the number of patients with PPA on your caseload has (please tick one box):

Increased

Decreased

Stayed the same

If increased or decreased, please explain why you think this has happened: 
14. Which professionals mainly refer people with PPA to the speech and language therapy service where you work? (tick all that apply)

Psychiatrists

Neurologists

Geriatricians

GPs

Psychologists

Other Speech and Language Therapists

Other:

15 What were the symptoms that prompted the referral to your service?

(Please estimate the percentage of referrals for each item)

Word finding difficulties

Slurred speech

Volume of speech

Swallowing difficulties

Other:

16. Prior to their referral to you, were the patients and their family members made aware of their diagnosis of PPA?

Always/mostly/sometimes/occasionally/never

17. If the patient/family members were NOT made aware of the diagnosis, what other terms / labels were used by the doctor to describe the condition, if any? (open box)

18. If the patient/family member have been made aware, which of these terms was used?

Primary progressive aphasia

Logopenic PPA

Semantic PPA

Non-fluent PPA

Semantic dementia 
Frontotemporal dementia

Other:

19. Were they informed that this was a type of dementia?

Always/mostly/sometimes/occasionally/never

20. What difficulties if any do patients report in getting a diagnosis of PPA? (open box?)

21. When are the majority of these patients being referred to you? (please tick one box)

Within a year of symptom onset (around diagnosis)

2-3 years post onset (mid-way through the disease process when language symptoms are still the primary issue)

$4+$ years post onset (later stage of the disease when cognitive symptoms have emerged)

Unknown

22. Do you have an established care pathway for people with PPA?

Yes/no

Please describe:

\section{Section 3: Time spent on management of this patient group}

23. Does your service stipulate how long an individual's intervention can last when they have a diagnosis of PPA? For example intermediate care often has a six-week intervention limit

Yes - Please tick the approximate number of sessions (please tick one)

1-2 sessions

2-3 sessions

4-6 sessions

$6+$ sessions

Other, please specify 
No - How many sessions on average would you estimate you spend with an individual from this patient group

Please tick the approximate number of sessions (please tick one)

1-2 sessions

2-3 sessions

4-6 sessions

$6+$ sessions

Other, please specify

24. Please estimate how many sessions you spend on the following when working with patients with PPA and / or their families

Direct assessment of language and communication

Assessment of dysphagia

Contributing to diagnosis of PPA

Impairment-directed language intervention

Functional interventions to support communication

Management of dysphagia

Education/information for patient

Education/information for family

Education/information for staff

Joint language / communication focused intervention with patient and family/friend/other

Mental Capacity assessment

Case/care review meetings

Joint sessions with other disciplines

Group therapy

25. If you are providing functional interventions to support communication, in what setting would these ideally occur in your opinion? (tick one)

Inpatient

Outpatient 


\section{Domiciliary}

26. Ideally how much time would you like to spend with someone with PPA on functional interventions to support communication?

Enter number of sessions:

\section{Section 4: Specific assessment and intervention approaches}

27. When working with people with PPA how often do you use each of the following assessments/tools?

Always/ often/sometimes/occasionally/never

Comprehensive Aphasia Test (CAT) - Swinburn, Porter \& Howard (2004)

Psycholinguistic Assessment of Language Processing in Aphasia (PALPA) Kay, Lesser \& Coltheart (1992)

Boston Naming Test

Cognitive Linguistic Quick Test (CLQT) - Helm-Estabrooks (2001)

Arizona Battery for Communication Disorders (ABCD) - Bayles \& Tomoeda (1993)

Barnes Language Assessment - Designed by Psychiatry of Old Age SIG members.

Mini Mental State Examination (MMSE)

Informal impairment based communication test/screen

Informal interview with person / family on personal history / conversation style / personality etc

Informal assessment or observation of communication in functional activities

If you sue any other assessment tools give name/authors (if appropriate), and a brief description of purpose

28. When working with people with PPA how often do you use the following intervention options?

Always/ often/sometimes/occasionally/never

Impairment-directed interventions including naming, semantic attributes, lexical retrieval, object use, phonological skills,

Impairment-directed interventions for speech production (apraxia of speech) 


\begin{abstract}
Work on reading and writing
Activity participation rehabilitation e.g. working on communication activities for a cooking task or TV watching Functional communication e.g. practising the use of multi-modal strategies such as gesture, drawing, writing

Communication training for families / carers

Communication training for staff

High tech communication aid provision and training- e.g. making an informed choice about app/device and training the client to use it, developing a communication book / word book or diary system etc

Introducing the person and their family to social and support networks
\end{abstract}

If you use any other interventions please describe

\title{
Section 5: Outcome measures and planning for the future
}

29. Do you use any of the following as an outcome measure for patients with PPA:

Goal setting

TOMS (ref)

Language assessments e.g. naming, picture description

Self-rating scales

Speech and language therapist rating scale

Video-recording of communication

Other. Please specify

30. Please provide one example of a goal that you have set with a patient with PPA. Open question:

31. Do you explicitly address any of the following in planning for the future with patients with PPA? Tick all that apply:

Cognitive changes and deterioration in communication

Legal, financial, health and social care issues, including assessment of capacity.

Driving

Family care supports such as respite

Family financial supports such as benefits

Other. Please specify: 
32. Do you refer patients and their families to any of the following charity or third sector organisations? Tick all that apply:

PPA support group

Alzheimer's Society

Dyscover

Stroke Association

Connect

Other - please specify 